\title{
Exploiting Isospin Symmetry to Study the Role of Isomers in Stellar Environments
}

\author{
S. Hallam, ${ }^{1}$ G. Lotay, ${ }^{1}$ A. Gade $\odot,{ }^{2,3,4}$ D. T. Doherty, ${ }^{1}$ J. Belarge, ${ }^{2}$ P. C. Bender, ${ }^{2, *}$ B. A. Brown, ${ }^{2,3,4}$ \\ J. Browne, ${ }^{2,3,4}$ W. N. Catford, ${ }^{1}$ B. Elman, ${ }^{2,3}$ A. Estradé, ${ }^{5}$ M. R. Hall, ${ }^{6}$ B. Longfellow, ${ }^{2,3}$ E. Lunderberg, ${ }^{2,3}$ \\ F. Montes, ${ }^{2,4}$ M. Moukaddam, ${ }^{1, \dagger}$ P. O’Malley, ${ }^{6}$ W.-J. Ong, ${ }^{2,3}$ H. Schatz, ${ }^{2,3,4}$ D. Seweryniak, ${ }^{7}$ \\ K. Schmidt $\odot,{ }^{2,4, \$}$ N. K. Timofeyuk $\odot,{ }^{1}$ D. Weisshaar $\odot,{ }^{2}$ and R. G. T. Zegers ${ }^{2,3,4}$ \\ ${ }^{1}$ Department of Physics, University of Surrey, Guildford GU2 7XH, United Kingdom \\ ${ }^{2}$ National Superconducting Cyclotron Laboratory, Michigan State University, East Lansing, Michigan 48824, USA \\ ${ }^{3}$ Department of Physics and Astronomy, Michigan State University, East Lansing, Michigan 48824, USA \\ ${ }^{4}$ Joint Institute for Nuclear Astrophysics, Center for the Evolution of the Elements, Michigan State University, \\ East Lansing, Michigan 48824, USA \\ ${ }^{5}$ Department of Physics, Central Michigan University, Mount Pleasant, Michigan 48859, USA \\ ${ }^{6}$ Department of Physics, University of Notre Dame, Notre Dame, Indiana 46556, USA \\ ${ }^{7}$ Physics Division, Argonne National Laboratory, Argonne, Illinois 60439, USA
}

(Received 23 October 2020; revised 23 November 2020; accepted 12 January 2021; published 29 January 2021)

\begin{abstract}
Proton capture on the excited isomeric state of ${ }^{26} \mathrm{Al}$ strongly influences the abundance of ${ }^{26} \mathrm{Mg}$ ejected in explosive astronomical events and, as such, plays a critical role in determining the initial content of radiogenic ${ }^{26} \mathrm{Al}$ in presolar grains. This reaction also affects the temperature range for thermal equilibrium between the ground and isomeric levels. We present a novel technique, which exploits the isospin symmetry of the nuclear force, to address the long-standing challenge of determining proton-capture rates on excited nuclear levels. Such a technique has in-built tests that strongly support its veracity and, for the first time, we have experimentally constrained the strengths of resonances that dominate the astrophysical ${ }^{26 m} \mathrm{Al}(p, \gamma)^{27} \mathrm{Si}$ reaction. These constraints demonstrate that the rate is at least a factor $\sim 8$ lower than previously expected, indicating an increase in the stellar production of ${ }^{26} \mathrm{Mg}$ and a possible need to reinvestigate sensitivity studies involving the thermal equilibration of ${ }^{26} \mathrm{Al}$.
\end{abstract}

DOI: 10.1103/PhysRevLett.126.042701

The radioisotope ${ }^{26} \mathrm{Al}$ provides us with rare insight into the nature of nuclear processes in stars throughout the Milky Way. Its existence in the early Solar System has been inferred from the observation of ${ }^{26} \mathrm{Mg}$ isotopic excesses in meteorites [1], while space-based observations of the characteristic $1.809-\mathrm{MeV} \gamma$ rays, associated with its $\beta$ decay, have provided direct confirmation of active nucleosynthesis in our Galaxy [2,3]. In fact, it has even been suggested that energy released by the in situ decay of ${ }^{26} \mathrm{Al}$ in protoplanetary disks, orbiting young stars, may have caused the melting of icy planetesimals, thereby influencing the conditions required of planetary systems to support life [4]. Consequently, determining the astrophysical origin of ${ }^{26} \mathrm{Al}$ represents one of the key goals of modern nuclear astrophysics.

Recently, large ${ }^{26} \mathrm{Al} /{ }^{27} \mathrm{Al}$ ratios have been reported for several presolar grains of possible nova and asymptotic giant branch (AGB) star origins [5-8], indicating that such environments may make a significant contribution to the overall galactic abundance of ${ }^{26} \mathrm{Al}$. However, observations of ${ }^{26} \mathrm{Al}$ cosmic $\gamma$ rays, by the COMPTEL and INTEGRAL satellite missions, point towards massive stars and corecollapse supernovae as being the likely dominant astrophysical source [9]. Hence, the exact origin of ${ }^{26} \mathrm{Al}$ remains controversial.
In this regard, stellar nucleosynthesis of ${ }^{26} \mathrm{Al}$ is complicated by the presence of a $0^{+}$isomer, ${ }^{26 m} \mathrm{Al}$ (half life $t_{1 / 2}=6.3 \mathrm{~s}$ ), located 228.31(3) $\mathrm{keV}$ above the $5^{+}$ground state, ${ }^{26 g} \mathrm{Al} \quad\left(t_{1 / 2}=7.2 \times 10^{5} \mathrm{yr}\right)$. This isomeric level undergoes a superallowed $\beta^{+}$decay directly to the ${ }^{26} \mathrm{Mg}$ ground state, bypassing emission of the telltale $1.809-\mathrm{MeV}$ $\gamma$ ray. As such, it does not contribute to the abundance of ${ }^{26} \mathrm{Al}$ inferred by space-based telescopes. However, reactions involving the isomeric state of ${ }^{26} \mathrm{Al}$ directly affect the astrophysical production of ${ }^{26} \mathrm{Mg}$, which needs to be understood in order to ascribe ${ }^{26} \mathrm{Al}$ signatures to presolar grains. Therefore, it is imperative that uncertainties in the production and destruction of ${ }^{26 m} \mathrm{Al}$ in stellar scenarios be reduced. Furthermore, while neutron-capture reactions are expected to have the most significant influence on the observed flux of ${ }^{26} \mathrm{Al} \gamma$ rays from supernovae, the relative proton-capture rates on ${ }^{26 g} \mathrm{Al}$ and ${ }^{26 m} \mathrm{Al}$ will determine the onset of thermal equilibrium between the two levels - this results in a reduction of the astrophysical half-life of ${ }^{26 g} \mathrm{Al}$ and impacts on the amount of ${ }^{26} \mathrm{Al}$ produced at high temperatures [10] of relevance for $\gamma$-ray observations.

At stellar temperatures below $0.1 \mathrm{GK},{ }^{26 m} \mathrm{Al}$ and ${ }^{26 g} \mathrm{Al}$ are produced in roughly equal quantities via the reaction 
sequence: ${ }^{24} \operatorname{Mg}(p, \gamma){ }^{25} \operatorname{Al}\left(\beta^{+} \nu_{e}\right){ }^{25} \operatorname{Mg}(p, \gamma)$ [11]. However, in higher-temperature scenarios $(T \geq 0.3 \mathrm{GK})$, such as oxygen-neon $(\mathrm{ONe})$ novae, the ${ }^{25} \mathrm{Al}(p, \gamma)^{26} \mathrm{Si}$ reaction competes significantly with the $\beta^{+}$decay of ${ }^{25} \mathrm{Al}$, and a large fraction of explosive hydrogen-burning events bypass the direct population of the ${ }^{26} \mathrm{Al}$ ground state $[12,13]$. As such, nuclear reactions on the isomer are likely to play a more significant role in these environments. Specifically, depending on the energies of excited states above the proton threshold of 7691.3(1) $\mathrm{keV}$ in ${ }^{27} \mathrm{Si}$ [14] and the proton occupation of orbitals (quantified here as a spectroscopic factor), isomeric-capture reactions may govern the pathway of nucleosynthesis in certain stellar scenarios.

In AGB stars $\left(T_{\text {peak }} \sim 0.14 \mathrm{GK}\right)$ and classical novae ( $\left.T_{\text {peak }} \sim 0.2-0.4 \mathrm{GK}\right)$, the destruction of ${ }^{26} \mathrm{Al}$ is governed by the ${ }^{26 g} \mathrm{Al}(p, \gamma)^{27} \mathrm{Si}$ and ${ }^{26 m} \mathrm{Al}(p, \gamma){ }^{27} \mathrm{Si}$ reactions, respectively. These reactions are dominated by resonant capture into excited states above the proton threshold in ${ }^{27} \mathrm{Si}$ and, over the past three decades, states of relevance for the ${ }^{269} \mathrm{Al}(p, \gamma)^{27} \mathrm{Si}$ reaction have been studied extensively [15-24]. In contrast, very little experimental information is available on the rate of the ${ }^{26 m} \mathrm{Al}(p, \gamma)^{27} \mathrm{Si}$ reaction [25-29]. This is due to the immense difficulty in producing beams of pure, isomeric ${ }^{26} \mathrm{Al}$ to uniquely probe low-spin excited states in ${ }^{27} \mathrm{Si}$. Previous estimates that have been used in astrophysical nuclear reaction network calculations are largely based on ${ }^{26 g} \mathrm{Al}+p$ resonances and HauserFeshbach calculations [30]. As such, these may be inappropriate for temperatures $\leq 0.4 \mathrm{GK}$, where the strengths of individual resonances are critical. In fact, a postprocessing study by Iliadis et al. [31] concluded that uncertainties in the ${ }^{26 m} \mathrm{Al}(p, \gamma)^{27} \mathrm{Si}$ reaction may affect the isotopic abundance of ${ }^{26} \mathrm{Mg}$ synthesized in novae environments by more than an order of magnitude.

One of the earliest attempts to experimentally investigate the ${ }^{26 m} \mathrm{Al}(p, \gamma)$ process [26] involved the use of ${ }^{27} \mathrm{Al}\left({ }^{3} \mathrm{He}, t\right){ }^{27} \mathrm{Si}(p){ }^{26} \mathrm{Al}$ and ${ }^{28} \mathrm{Si}\left({ }^{3} \mathrm{He}, \alpha\right){ }^{27} \mathrm{Si}(p){ }^{26} \mathrm{Al}$ reactions to observe proton decays from excited states above the ${ }^{26 m} \mathrm{Al}+p$ threshold. In that study [26], an excited state at 8136(4) $\mathrm{keV}$, corresponding to a resonance in the ${ }^{26 m} \mathrm{Al}+p$ system at $E_{r}=445(4) \mathrm{keV}$, was found to dominate the stellar reaction rate. However, $445 \mathrm{keV}$ also represented the energy threshold cutoff of the detection system and as such, it was not possible to observe any resonances with $E_{r}<445 \mathrm{keV}$. This is of particular significance since the Gamow window for the ${ }^{26 m} \mathrm{Al}(p, \gamma)$ reaction in AGB stars and classical novae covers an energy range of $E_{r} \sim 100-500 \mathrm{keV}$. Consequently, following the pioneering work of Deibel et al. [26], a spectroscopy study of ${ }^{27} \mathrm{Si}$ was performed to identify low-energy ${ }^{26 m} \mathrm{Al}+p$ resonant states by their $\gamma$ decays $[27,28]$. That work [27,28], assigned a spin of $J=1 / 2$ to the resonance at $E_{r}=447.7(6) \mathrm{keV}$, supporting the results of Deibel et al. [26], and identified a $J=5 / 2$ resonance at $E_{r}=146.3(3) \mathrm{keV}$, as well as three $J=3 / 2$ resonances at $E_{r}=217.8(7), 378.3(30)$, and $492.2(4) \mathrm{keV}$, respectively. Parities were assigned, based on comparisons with the mirror nucleus, ${ }^{27} \mathrm{Al}$, and it was proposed that the $5 / 2^{+}$, $146 \mathrm{keV}$ resonance dominated the ${ }^{26 m} \mathrm{Al}(p, \gamma)$ reaction for $T<0.15 \mathrm{GK}$, while the $3 / 2^{-}, 378-\mathrm{keV}$ resonance determined the rate for $T \geq 0.2 \mathrm{GK}$. Unfortunately, despite these developments, the strengths of the resonances remain almost entirely unknown, leaving considerable uncertainty in the ${ }^{26 m} \mathrm{Al}(p, \gamma)$ stellar reaction rate. Moreover, conflicting information from subsequent studies $[29,32]$ has obfuscated the parity of the $218-\mathrm{keV}$ resonance, while a measurement of the ${ }^{28} \mathrm{Si}\left({ }^{3} \mathrm{He}, \alpha\right)$ reaction now indicates a $3 / 2^{+}$reassignment for the $378-\mathrm{keV}$ state [29].

In this Letter, we present a novel method for investigating ${ }^{26 m} \mathrm{Al}+p$ resonances using the ${ }^{26} \mathrm{Si}(d, p)$ transfer reaction. In particular, we capitalize on the elegant concept of isospin symmetry, which allows us to describe the structures of nuclear states with the same isospin with the exact same wave function. Here, the nucleus ${ }^{26} \mathrm{Si}$ forms part of a $0^{+}$isobaric triplet, ${ }^{26} \mathrm{Si}-{ }^{26 m} \mathrm{Al}-{ }^{26} \mathrm{Mg}$, and, as such, neutron transfer on ${ }^{26} \mathrm{Si}$ acts as a surrogate for the astrophysical ${ }^{26 m} \mathrm{Al}(p, \gamma){ }^{27} \mathrm{Si}$ reaction. This provides several distinct advantages over previous studies. Specifically, the reaction mechanism directly populates low-spin excited states of interest in ${ }^{27} \mathrm{Si}$, while also eliminating any background associated with the ${ }^{26} \mathrm{Al}$ ground state. Furthermore, spectroscopic factors, $C^{2} S$, extracted for levels in ${ }^{27} \mathrm{Si}$, from neutron transfer on ${ }^{26} \mathrm{Si}$, are inherently twice as large as those for proton capture on ${ }^{26 m} \mathrm{Al}$, owing to the associated Clebsch-Gordon coefficients. This feature is not only useful to improve the accuracy of $C^{2} S$ but also allows for greater sensitivity in the assignment of upper limits for unobserved excited states. Here, states in ${ }^{27} \mathrm{Si}$ were identified by their characteristic $\gamma$ decays [27,28] and angle-integrated cross sections were derived from observed $\gamma$-ray intensities, as well as known branching ratios of analog states in the mirror nucleus, ${ }^{27} \mathrm{Al}$ [33]. This methodology has previously been successfully employed in studies of the ${ }^{26} \mathrm{Al}(d, n)$ [23] and ${ }^{30} \mathrm{P}(d, n)$ [34] reactions.

A beam of radioactive ${ }^{26} \mathrm{Si}$ ions at $30 \mathrm{MeV} / \mathrm{u}$ was produced by projectile fragmentation of a $150 \mathrm{MeV} / \mathrm{u}$ primary beam of ${ }^{36} \mathrm{Ar}$ ions at the National Superconducting Cyclotron Laboratory. This beam was then used to bombard a $9.1(7)-\mathrm{mg} / \mathrm{cm}^{2}$-thick deuterated polyethylene target $\left(\mathrm{CD}_{2}\right)_{n}$, with a typical intensity of $\sim 1 \times 10^{5} \mathrm{pps}$. Two time-of-flight measurements between a series of fast plastic scintillators (two upstream of the target and one downstream) allowed for the identification of incoming ${ }^{26} \mathrm{Si}$ particles, event by event. The ${ }^{26} \mathrm{Si}$ beam purity was determined to be $60(5) \%$ and ${ }^{25} \mathrm{Al}$ was found to represent the main contaminant species. Prompt $\gamma$ rays were observed with the GRETINA tracking array [35], which, in this instance, comprised of 10 modules positioned at laboratory angles of $58^{\circ}$ and $90^{\circ}$, respectively, while projectilelike 
reaction products were transmitted to the focal plane of the S800 spectrograph [36]. Extracted cross sections for lowlying excited states in ${ }^{27} \mathrm{Si}$, populated via transfer, may be overestimated due to the indirect feeding of states, by discrete $\gamma$-ray transitions, from higher-lying levels. As such, a $\gamma-\gamma$ coincidence matrix was produced in order to quantify the relative feeding of levels in ${ }^{27} \mathrm{Si}$ and remove such counts from the calculations of cross sections. The S800 spectrometer was run in focused mode and provided clean separation of ${ }^{27} \mathrm{Si}$ ions from other recoil species, as well as a $95(1) \%$ acceptance. The GRETINA efficiency was obtained from a GEANT 4 simulation and validated by source measurements covering energies up to $3.5 \mathrm{MeV}$, as well as high-energy $\gamma$-ray yields up to $6 \mathrm{MeV}$, produced in beam by nucleon-removal reactions on ${ }^{18} \mathrm{O}$. Finally, the possibility of background generated by ${ }^{26} \mathrm{Si}\left({ }^{12} \mathrm{C},{ }^{11} \mathrm{C}\right)$ reactions was investigated using a 13(1)-mg/ $\mathrm{cm}^{2}$-thick polyethylene target $\left(\mathrm{CH}_{2}\right)_{n}$. However, the level of background was found to be negligible.

Figure 1 shows the Doppler-corrected $\gamma$-ray spectrum measured with GRETINA when gating on ${ }^{27} \mathrm{Si}$ ions at the focal plane of the S800 spectrograph. The selective population of low-spin excited states in ${ }^{27} \mathrm{Si}$ via the ${ }^{26} \mathrm{Si}(d, p)$ reaction is clearly highlighted. However, no $\gamma$ decays were observed from any proton-unbound excited levels and, as such, it is expected that no strong single-particle states exist within the resonance energy region, $E_{r}=100-500 \mathrm{keV}$, in the ${ }^{26 m} \mathrm{Al}+p$ system. Nevertheless, the absence of significant background makes it possible to assign stringent upper limits for the strengths of these resonances based on the present data. Spectroscopic factors were extracted by comparing measured cross sections to theoretical values obtained from calculations in the adiabatic distorted wave approximation
(ADWA) using the code TWOFNR [37]. Here, the KoningDelaroche global optical model parameterization [38] was used to calculate the $d-{ }^{26} \mathrm{Si}$ distorting potentials [39]. Table I presents a summary of extracted spectroscopic factors for observed levels in ${ }^{27} \mathrm{Si}$, together with a comparison to analog states in ${ }^{27} \mathrm{Al}$ and shell-model calculations. Shell-model calculations are based on the USDB Hamiltonian within the $s d$-shell model space [40] for evenparity states, and on the WBP Hamiltonian, which includes a $s d-p f$ Hamiltonian, for odd-parity states [41].

In general, good agreement is observed between the spectroscopic factors $C^{2} S$ of excited states in ${ }^{27} \mathrm{Si}$ and those of analog states in ${ }^{27} \mathrm{Al}$, determined via mirror ${ }^{26} \mathrm{Mg}(d, n)$ and ${ }^{26} \mathrm{Mg}\left({ }^{3} \mathrm{He}, d\right)$ reactions. Moreover, the present results are reasonably well reproduced by shell-model calculations. For completeness, we note that excited levels in ${ }^{27} \mathrm{Si}$ at 5850 and $6559 \mathrm{keV}$ were previously assigned as $3 / 2^{+}$ states [46]. However, the presently reported cross sections would result in unfeasibly large $C^{2} S$ for transfers to the $1 d_{3 / 2}$ orbital. Consequently, assignments of $\left(3 / 2^{-}, 7 / 2^{+}\right)$ and $3 / 2^{-}$are now indicated for the 5850- and $6559-\mathrm{keV}$ states, respectively. Furthermore, we report the observation of a high-energy excited state at $7262 \mathrm{keV}$ that, based on its measured cross section and comparison to shell-model calculations, most likely corresponds to the tentative $7 / 2^{-}$ state observed in the earlier work of Parikh et al. [29]. Intriguingly, it should be pointed out that the expected $C^{2} S$ of $\sim 0.2$, based on shell-model calculations, for the first $1 / 2^{+}$excited state in the ${ }^{27} \mathrm{Si}^{27} \mathrm{Al}$ system, populated via single-proton or single-neutron transfer on ${ }^{26 m} \mathrm{Al}$, is in disagreement with the recently reported value of $0.08(2)$ from a study of the ${ }^{26 m} \mathrm{Al}(d, p)$ reaction [25]. This discrepancy is possibly due to complications associated with the subtraction of background arising from the ${ }^{26} \mathrm{Al}$

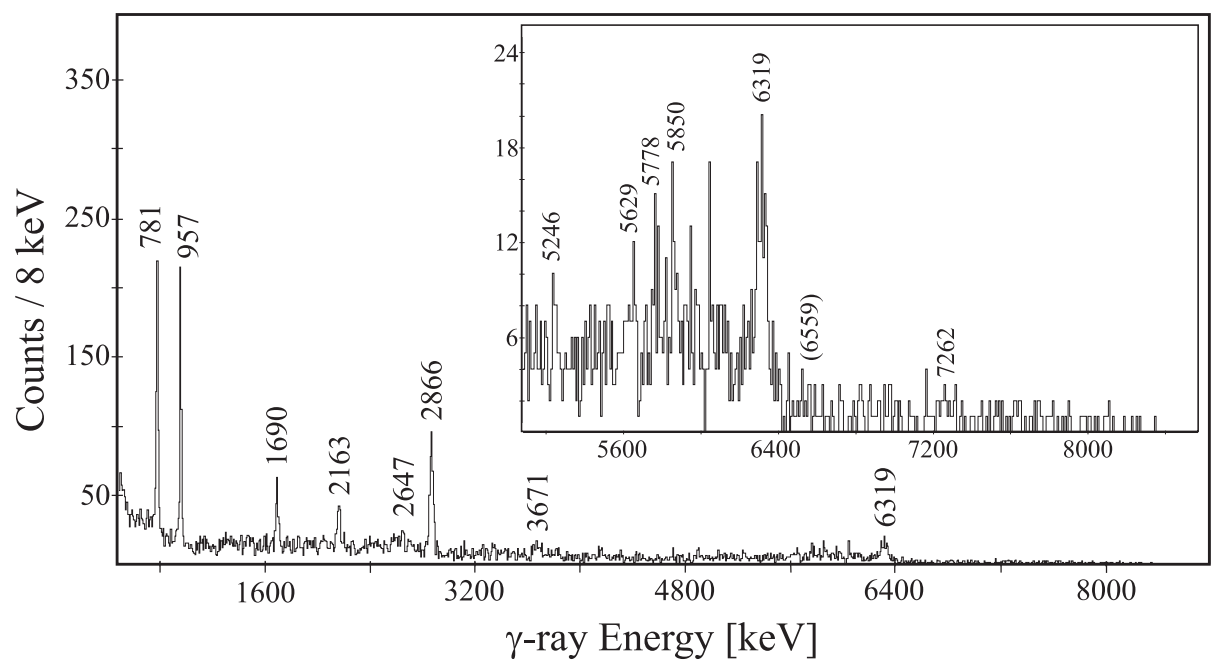

FIG. 1. Doppler-reconstructed $\gamma$-ray spectrum in coincidence with ${ }^{27} \mathrm{Si}$ recoils detected in the S800 spectrograph. (Inset) Expanded energy region of interest for nuclear astrophysics. A low-intensity transition is observed at $7262 \mathrm{keV}$. Fitting the peak based on the width of the $6319-\mathrm{keV} \gamma$ ray yields a total of 13 counts, supporting its existence at the $\sim 2 \sigma$ confidence level, assuming a flat background. 
TABLE I. Properties of states in ${ }^{27} \mathrm{Si}$ populated in the ${ }^{26} \mathrm{Si}(d, p)$ reaction. Excitation energies and $J^{\pi}$ assignments are adopted from Ref. [27] unless noted otherwise, and spectroscopic factors, $C^{2} S$, for analog states in ${ }^{27} \mathrm{Al}$ have been taken from ${ }^{26} \mathrm{Mg}(d, n)[42]$ and ${ }^{26} \mathrm{Mg}\left({ }^{3} \mathrm{He}, d\right)$ [43-45] reaction studies. The values $C^{2} S_{\mathrm{SM}}$ relate to shell-model calculations for the ${ }^{26} \mathrm{Si}(d, p)$ reaction.

\begin{tabular}{|c|c|c|c|c|c|c|c|c|c|c|}
\hline$E_{x}$ & $E_{\gamma}$ & $B . R$. & $J_{n}^{\pi}$ & $\sigma$ & $n l j$ & $C^{2} S_{(d, p)}$ & $C^{2} S_{(d, n)}$ & $C^{2} S_{\left({ }^{3} \mathrm{He}, d\right)}$ & $C^{2} S_{\mathrm{SM}}$ & Analog state in ${ }^{27} \mathrm{Al}$ \\
\hline & {$[\mathrm{keV}]$} & $\%$ & & {$[\mu b]$} & ADWA & & [42] & {$[43-45]$} & & {$[\mathrm{keV}]$} \\
\hline 781 & 781 & 100 & $1 / 2_{1}^{+}$ & $780(170)$ & $2 s_{1 / 2}$ & $0.43(9)$ & 0.41 & 0.50 & 0.41 & 844 \\
\hline 957 & 957 & 94 & $3 / 2_{1}^{+}$ & $610(140)$ & $1 d_{3 / 2}$ & $0.11(3)$ & 0.08 & 0.07 & 0.05 & 1014 \\
\hline 2163 & 2163 & 100 & $7 / 2_{1}^{+}$ & $440(110)$ & & & & & & 2212 \\
\hline 2647 & $\begin{array}{l}1690 \\
2647\end{array}$ & $\begin{array}{l}77 \\
20\end{array}$ & $5 / 2_{2}^{+}$ & $300(90)$ & $1 d_{5 / 2}$ & $0.05(2)$ & 0.03 & 0.04 & 0.007 & 2735 \\
\hline 2866 & 2866 & 96 & $3 / 2_{2}^{+}$ & $1790(390)$ & $1 d_{3 / 2}$ & $0.38(8)$ & 0.47 & 0.63 & 0.32 & 2982 \\
\hline 4285 & $\begin{array}{l}3328 \\
4285\end{array}$ & $\begin{array}{l}41 \\
52\end{array}$ & $5 / 2_{3}^{+}$ & $380(140)$ & $1 d_{5 / 2}$ & $0.06(2)$ & 0.03 & 0.04 & 0.04 & 4410 \\
\hline 5850 & $\begin{array}{l}4893 \\
5850\end{array}$ & $\begin{array}{l}26 \\
74\end{array}$ & $\left(3 / 2_{2}^{-}\right)$ & 491(130) & $2 p_{3 / 2}$ & $0.06(2)$ & $\mathrm{a}$ & & 0.001 & $(6080)$ \\
\hline 5850 & $\begin{array}{l}4893 \\
5850\end{array}$ & $\begin{array}{l}26 \\
74\end{array}$ & $\left(7 / 2_{4}^{+}\right)$ & & & & $\mathrm{a}$ & & & $(5961)$ \\
\hline 6027 & 5246 & 80 & $3 / 2_{3}^{-}$ & $170(80)$ & $2 p_{3 / 2}$ & $0.02(1)$ & 0.02 & 0.04 & 0.09 & 6159 \\
\hline 6319 & $\begin{array}{l}3671 \\
4156 \\
6319\end{array}$ & $\begin{array}{c}15 \\
9 \\
76\end{array}$ & $7 / 2_{2}^{-}$ & $2850(650)$ & $1 f_{7 / 2}$ & $0.14(3)$ & 0.22 & 0.20 & 0.23 & 6477 \\
\hline 6559 & $\begin{array}{c}5778 \\
(6559)\end{array}$ & $\begin{array}{l}50 \\
17\end{array}$ & $3 / 2^{-} \mathrm{b}$ & $550(200)$ & $2 p_{3 / 2}$ & $0.07(3)$ & 0.07 & & 0.12 & 6604 \\
\hline 6586 & 5629 & 100 & $5 / 2_{8}^{+}$ & $200(60)$ & $1 d_{5 / 2}$ & $0.04(1)$ & $\mathrm{a}$ & & 0.002 & (6767) \\
\hline $7262^{c}$ & 7262 & 100 & $\left(7 / 2_{3}^{-}\right)$ & $160(60)$ & $1 f_{7 / 2}$ & $0.008(3)$ & $\mathrm{a}$ & & 0.002 & (7477) \\
\hline
\end{tabular}

${ }^{\mathrm{a}}$ Seen in Ref. [42] but no $C^{2} S$ reported.

${ }^{\mathrm{b}}$ Parity assignment based on extracted cross section.

${ }^{\mathrm{c}}$ State reported in Ref. [29].

ground state [25] and further highlights the benefit of using an analog reaction to directly populate the same states in ${ }^{27} \mathrm{Si}$, that are populated in the astrophysical ${ }^{26 m} \mathrm{Al}(p, \gamma)$ reaction.

For an evaluation of the ${ }^{26 m} \mathrm{Al}(p, \gamma)$ reaction rate, we consider only the contributions of the 146-, 218-, 378-, $448-$, and $492-\mathrm{keV}$ resonances. The $\gamma$-decay properties of these states have already been reported elsewhere $[27,46]$ and we assume, here, that they correspond to $\sim 100 \%$ branches (any significant low-energy branches would have been observed in Refs. [27,46]). Using the known $\gamma$-ray energies of Refs. [27,46], we have determined an upper limit for the angle-integrated cross section of each resonance. This was achieved by fixing the peak widths of transitions, based on the 6319- and 7262-keV $\gamma$ rays, and assessing the total number of counts present over the relevant energy regions of unobserved decays. While some background is expected in the regions investigated, we chose to base our upper limits on the total number of counts in order to obtain a far more conservative estimate. These cross sections enable the extraction of spectroscopic factors $C^{2} S$ for key resonant states in the ${ }^{26 m} \mathrm{Al}(p, \gamma)$ reaction and thus provide stringent experimental constraints on the rate [47]. We estimate an overall uncertainty of $\sim 36 \%$ in the determination of spectroscopic factors (based on earlier experimental work [23,34] and incorporating a 20\% uncertainty in the extraction of cross sections, due to possible unobserved $\gamma$-decay branches). In addition, we estimate a $\sim 35 \%$ uncertainty in shell-model calculations of $\gamma$-ray partial widths and a factor $\sim 1.7$ uncertainty in the derived proton-partial widths of resonant states [47]. In the case of the ${ }^{26 m} \mathrm{Al}+p$ resonant states at 218 and $448 \mathrm{keV}$, for which the parity remains ambiguous, we have determined $C^{2} S$ for both possibilities. Unfortunately, due to the high excitation energy of ${ }^{26 m} \mathrm{Al}+p$ resonant states, it is generally not possible to uniquely identify resonances with shell-model states. That being said, should the $448-\mathrm{keV}$ resonance correspond to a $1 / 2^{+}$state, it may be unambiguously identified as the $1 / 2_{7}^{+}$level, owing to the low leveldensity of $1 / 2^{+}$states in ${ }^{27} \mathrm{Si}$. As such, in estimating the $1 / 2^{+}, 448-\mathrm{keV}$ resonance strength, we have adopted a value of $C^{2} S=0.01$, from shell-model calculations. A summary of the properties of ${ }^{26 m} \mathrm{Al}(p, \gamma)$ resonances is presented in Table II.

Figure 2 illustrates the contribution of individual resonances to the ${ }^{26 m} \mathrm{Al}(p, \gamma)$ stellar reaction rate, together with a comparison of the current upper limit, accounting for uncertainties, to the earlier REACLIB estimate [48], which, for temperatures $\leq 0.4 \mathrm{GK}$, is based on the NACRE evaluation [30]. The new upper limit obtained in this work indicates that the ${ }^{26 m} \mathrm{Al}(p, \gamma)$ reaction rate is at least a factor $\sim 8$ smaller than previously expected up to temperatures of 
TABLE II. Properties of resonant states in the ${ }^{26 m} \mathrm{Al}(p, \gamma)^{27} \mathrm{Si}$ reaction. Upper limits for proton partial widths have been estimated using present cross sections, while $\gamma$-ray widths have been determined using a lifetime lower limit of $1 f s$, for $\gamma$-decaying states, unless otherwise noted. In the case of the 218- and 448-keV resonances, we present strength estimates for both even- and odd-parity assignments.

\begin{tabular}{cccccccccc}
\hline \hline$E_{x}$ & $E_{\gamma}[27]$ & $E_{r}[27]$ & $J^{\pi}$ & $\sigma$ & $C^{2} S_{26} \operatorname{Si}(d, p){ }^{\mathrm{a}}$ & $C^{2} S_{26 m} \mathrm{Al}(p, \gamma)$ & ${ }^{\mathrm{a}}$ & $\Gamma_{p}$ & $\Gamma_{\gamma}$ \\
\hline & {$[\mathrm{keV}]$} & {$[\mathrm{keV}]$} & & {$[\mu b]$} & & & & {$[\mathrm{meV}]$} & {$[\mathrm{meV}]$} \\
\hline 7838 & $6879.6(2)$ & $146.3(3)$ & $5 / 2^{+}$ & $\leq 168$ & $\leq 0.03$ & $\leq 0.015$ & $\leq 4.9 \times 10^{-6}$ & $\leq 658$ & $\leq 1.5 \times 10^{-5}$ \\
7909 & $7127.1(7)$ & $217.8(7)$ & $3 / 2^{-}$ & $\leq 43$ & $\leq 0.01$ & $\leq 0.005$ & $\leq 2.7 \times 10^{-2}$ & $\leq 658$ & $\leq 0.054$ \\
& & & $3 / 2^{+}$ & & $\leq 0.01$ & $\leq 0.005$ & $\leq 7.1 \times 10^{-4}$ & $\leq 658$ & $\leq 1.4 \times 10^{-3}$ \\
8070 & $7111.5(30)$ & $378.3(30)$ & $3 / 2^{+}$ & $\leq 14$ & $\leq 0.003$ & $\leq 0.0015$ & $\leq 0.16$ & $\leq 658$ & $\leq 0.33$ \\
8140 & $7180.9(6)$ & $447.7(6)$ & $1 / 2^{+}$ & $\leq 58$ & $\leq 0.09$ & $\leq 0.045$ & $683^{\mathrm{b}}$ & $890^{\mathrm{c}}$ & 385 \\
& & & $1 / 2^{-}$ & & $\leq 0.02$ & $\leq 0.01$ & $\leq 190$ & $\leq 658$ & $\leq 147$ \\
8184 & $7401.7(4)$ & $492.2(4)$ & $3 / 2^{-}$ & $\leq 15$ & $\leq 0.002$ & $\leq 0.001$ & $\leq 45$ & $165[27]$ & $\leq 70$ \\
\hline \hline
\end{tabular}

${ }^{a} C_{(d, p)}^{2}=2 / 3$ and $C_{(p, \gamma)}^{2}=1 / 3$

$\left.{ }^{\mathrm{b}} C^{2} S^{2}\right)=0.01-$ see text for details

${ }^{c}$ Adopted from shell-model calculations for the $1 / 2_{7}^{+}$state

0.35 GK. We therefore conclude that there will be an increase in the expected abundance of ${ }^{26} \mathrm{Mg}$ synthesized in AGB stars and classical nova explosions. In particular, the nova sensitivity study of Iliadis et al. [31], predicts that our new rate will lead to a $30 \%-60 \%$ increase in the ejected

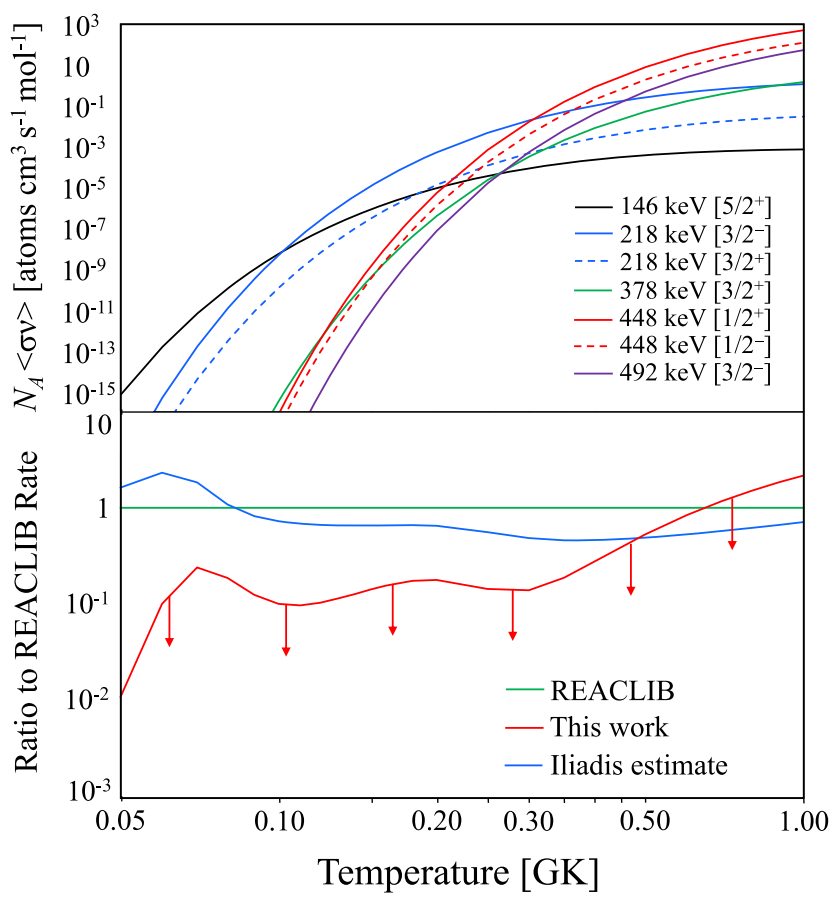

FIG. 2. (top) Upper-limit contributions of individual resonances to the ${ }^{26 m} \mathrm{Al}(p, \gamma)$ stellar reaction rate. The direct capture component for the ${ }^{26 m} \mathrm{Al}(p, \gamma)$ reaction is negligible for temperatures $>0.02 \mathrm{GK}$ [17]. (bottom) Comparison of the ${ }^{26 m} \mathrm{Al}(p, \gamma)$ rate from this work, accounting for systematic uncertainties stated in the text, with the previously reported REACLIB estimate [48], and the experimentally constrained ${ }^{26 g} \mathrm{Al}(p, \gamma)$ reaction, which has been recommended as an approximation for ${ }^{26 m} \mathrm{Al}(p, \gamma)$ [49]. abundance of ${ }^{26} \mathrm{Mg}$ in nova events. Consequently, the information obtained here is critical for accurately classifying the initial ${ }^{26} \mathrm{Al} /{ }^{27} \mathrm{Al}$ ratio in presolar grains and hence, the contribution of AGB stars and classical novae to the observed galactic abundance of ${ }^{26} \mathrm{Al}$. Furthermore, it is also fascinating to note that the current ${ }^{26 m} \mathrm{Al}(p, \gamma)$ rate is considerably slower than the corresponding ${ }^{26 g} \mathrm{Al}(p, \gamma)$ reaction, which has recently been suggested as a more reliable approximation for the ${ }^{26 m} \mathrm{Al}(p, \gamma)$ rate [49]. Given the significant discrepancy between the current rate and theoretical calculations [48], we would encourage that sensitivity studies of the destruction of ${ }^{26} \mathrm{Al}$ in massive stars and CCSN be repeated-although it is currently anticipated that the equilibration of ${ }^{26} \mathrm{Al}$ levels has only a minor effect on the ${ }^{26} \mathrm{Al}$ yields in massive star sites [49].

In summary, we have, for the first time, been able to mimic isomeric-state proton capture in astrophysical environments, utilizing isospin symmetry. In particular, the nonobservation of electromagnetic transitions from excited states in ${ }^{27} \mathrm{Si}$, known to predominantly $\gamma$ decay [27], demonstrates that there are no strong, single-particle states in the energy region $E_{r}=100-500 \mathrm{keV}$ of the ${ }^{26 m} \mathrm{Al}(p, \gamma)$ reaction. As such, the stellar reaction rate is significantly lower than previously expected $[30,48,49]$, over the temperature range $\sim 0.05-0.35 \mathrm{GK}$. We now expect a considerable increase in the ejected abundance of ${ }^{26} \mathrm{Mg}$ from $\mathrm{AGB}$ stars and classical novae, and highlight a possible need to revisit sensitivity studies of the thermal equilibration of ${ }^{26} \mathrm{Al}$. Furthermore, with its proven versatility, the present, pioneering technique may be extended to investigations of other astrophysically important processes involving isomeric states, such as the ${ }^{34 g, m} \mathrm{Cl}(p, \gamma)$ and ${ }^{38 g, m} \mathrm{~K}(p, \gamma)$ reactions.

This work was supported by U.S. National Science Foundation (NSF) under Grants No. PHY-1565546 (NSCL), No. PHY-1913554, No. PHY-1811855, and 
No. PHY-1430152 (JINA-CEE), the DOE National Nuclear Security Administration through the Nuclear Science and Security Consortium, under Award No. DE-NA0003180, and by the U.S. Department of Energy, Office of Science, Office of Nuclear Physics, under Grants No. DESC0020451 (MSU). GRETINA was funded by the U.S. Department of Energy, Office of Science. Operation of the array at N. S. C. L. was supported by the DOE under Grants No. DE-SC0014537 (NSCL) and No. DE-AC0205CH11231 (LBNL). UK personnel were supported by the Science and Technologies Facilities Council (STFC).

*Present address: Department of Physics, University of Massachusetts Lowell, Lowell, Massachusetts 01854, USA. †Present address: Université de Strasbourg, CNRS, IPHC UMR 7178, F-67000 Strasbourg, France.

*Present address: Institute of Radiation Physics, HelmholtsZentrum Dresden-Rossendorf, Bautzner Landstraße 400, 01328 Dresden, Germany.

[1] G. J. MacPherson, A. M. Davies, and E. K. Zinner, Meteoritics 30, 365 (1995).

[2] W. A. Mahoney, J. Ling, A. Jacobson, and R. Lingenfelter, Astrophys. J. 262, 742 (1982).

[3] R. Diehl et al., Astron. Astrophys. 298, 445 (1995), https:// ui.adsabs.harvard.edu/abs/1995A\%26A...298..445D/abstract.

[4] G. Srinivasan, J. N. Goswami, and N. Bhandari, Science 284, 1348 (1999).

[5] L. Nittler, C. M. O'D. Alexander, X. Gao, R. M. Walker, and E. Zinner, Astrophys. J. 483, 475 (1997).

[6] S. Amari, X. Gao, L. R. Nittler, E. Zinner, J. Jose, M. Hernanz, and R. S. Lewis, Astrophys. J. 551, 1065 (2001).

[7] L. Siess and M. Arnould, Astron. Astrophys. 489, 395 (2008).

[8] M. Bose and S. Starrfield, Astrophys. J. 873, 14 (2019).

[9] R. Diehl et al., Nature (London) 439, 45 (2006).

[10] R. C. Runkle, A. E. Champagne, and J. Engel, Astrophys. J. 556, 970 (2001).

[11] A. Straniero et al., Astrophys. J. 763, 100 (2013).

[12] M. B. Bennett et al., Phys. Rev. Lett. 111, 232503 (2013).

[13] P. N. Peplowski et al., Phys. Rev. C 79, 032801(R) (2009).

[14] M. Wang, G. Audi, A. H. Wapstra, F. G. Kondev, M. MacCormick, X. Xu, and B. Pfeiffer Chin. Phys. C 36, 1603 (2012).

[15] L. Buchmann, M. Hilgemeier, A. Krauss, A. Redder, C. Rolfs, H. P. Trautvetter, and T. R. Donoghue, Nucl. Phys. A415, 93 (1984).

[16] T. F. Wang, A. E. Champagne, J. D. Hadden, P. V. Magnus, M. S. Smith, A. J. Howard, and P. D. Parker, Nucl. Phys. A499, 546 (1989).

[17] A. E. Champagne, B. A. Brown, and R. Sherr, Nucl. Phys. A556, 123 (1993).

[18] R. B. Vogelaar, L. W. Mitchell, R. W. Kavanagh, A. E. Champagne, P. V. Magnus, M. S. Smith, A. J. Howard, P. D. Parker, and H. A. O'Brien, Phys. Rev. C 53, 1945 (1996).
[19] C. Ruiz et al., Phys. Rev. Lett. 96, 252501 (2006).

[20] G. Lotay, P. J. Woods, D. Seweryniak, M. P. Carpenter, R. V. F. Janssens, and S. Zhu Phys. Rev. Lett. 102, 162502 (2009).

[21] V. Margerin et al., Phys. Rev. Lett. 115, 062701 (2015).

[22] S. D. Pain et al., Phys. Rev. Lett. 114, 212501 (2015).

[23] A. Kankainen et al., Eur. Phys. J. A 52, 6 (2016).

[24] G. Lotay et al., Eur. Phys. J. A 56, 3 (2020).

[25] S. Almaraz-Calderon et al., Phys. Rev. Lett. 119, 072701 (2017).

[26] C. M. Deibel, J. A. Clark, R. Lewis, A. Parikh, P. D. Parker, and C. Wrede, Phys. Rev. C 80, 035806 (2009).

[27] G. Lotay, P. J. Woods, D. Seweryniak, M. P. Carpenter, R. V. F. Janssens, and S. Zhu, Phys. Rev. C 80, 055802 (2009).

[28] G. Lotay, P. J. Woods, D. Seweryniak, M. P. Carpenter, R. V. F. Janssens, and S. Zhu, Phys. Rev. C 81, 029903(E) (2010).

[29] A. Parikh et al., Phys. Rev. C 84, 065808 (2011).

[30] C. Angulo et al., Nucl. Phys. A656, 3 (1999).

[31] C. Iliadis, A. E. Champagne, J. José, S. Starrfield, and P. Tupper, Astrophys. J. Suppl. Ser. 142, 105 (2002).

[32] E. McCleskey et al. Phys. Rev. C 94, 065806 (2016).

[33] M. S. Basunia, Nucl. Data Sheets 112, 1875 (2011).

[34] A. Kankainen et al., Phys. Lett. B 769, 549 (2017).

[35] S. Paschalis et al., Nucl. Instrum. Methods Phys. Res., Sect. A 709, 44 (2013).

[36] D. Bazin, J. A. Caggiano, B. M. Sherrill, J. Yurkon, and A. Zeller, Nucl. Instrum. Methods Phys. Res., Sect. B 204, 629 (2003).

[37] J. A. Tostevin, University of Surrey version of the code TWOFNR (of M. Toyama, M. Igarashi, and N. Kishida) and code FRONT (private communication).

[38] A. J. Koning and J. P. Delaroche, Nucl. Phys. A713, 231 (2003).

[39] R. C. Johnson and P. C. Tandy, Nucl. Phys. A235, 56 (1974).

[40] B. A. Brown and W. A. Richter, Phys. Rev. C 74, 034315 (2006).

[41] E. K. Warburton and B. A. Brown, Phys. Rev. C 46, 923 (1992).

[42] J. Uzureau, A. Adam, and S. Joly, Nucl. Phys. A250, 163 (1975).

[43] W. Bohne et al., Nucl. Phys. A131, 273 (1969).

[44] H. F. Lutz, D. W. Heikkinen, W. Bartolini, and T. H. Curtis, Phys. Rev. C 2, 981 (1970).

[45] W. P. Alford, D. Cline, H. E. Gove, K. H. Purser, and S. Skorka, Nucl. Phys. A130, 119 (1969).

[46] G. Lotay, P. J. Woods, D. Seweryniak, M. P. Carpenter, H. M. David, R. V. F. Janssens, and S. Zhu, Phys. Rev. C 84, 035802 (2011).

[47] C. Iliadis, P. M. Endt, N. Prantzos, and W. J. Thompson, Astrophys. J. 524, 434 (1999).

[48] R. H. Cyburt et al., Astrophys. J. Suppl. Ser. 189, 240 (2010).

[49] C. Iliadis et al., Astrophys. J. Suppl. Ser. 193, 16 (2011). 